\title{
ASYMPTOTICS OF EIGENVALUES OF INFINITE BLOCK MATRICES
}

\author{
I.N. BRAEUTIGAM，D.M. POLYAKOV
}

\begin{abstract}
The paper is devoted to determining the asymptotic behavior of eigenvalues, which is one of topical directions in studying operators generated by tridiagonal infinite block matrices in Hilbert spaces of infinite sequences with complex coordinates or, in other words, to discrete Sturm-Liouville operators. In the work we consider a class of non-selfadjoint operators with discrete spectrum being a sum of a self-adjoint operator serving as an unperturbed operator and a perturbation, which is an operator relatively compact with respect to the unperturbed operator. In order to study the asymptotic behavior of eigenvalues, in the paper we develop an adapted scheme of abstract method of similar operators. The main idea of this approach is that by means of the similarity operator, the studying of spectral properties of the original operator is reduced to studying the spectral properties of an operator of a simpler structure. Employing this scheme, we write out the formulae for the asymptotics of arithmetical means of the eigenvalues of the considered class of the operators. We note that such approach differs essentially from those employed before. The obtained general result is applied for determining eigenvalues of particular operators. Namely, we provide asymptotics for the eigenvalues of symmetric and non-symmetric tridiagonal infinite matrices in the scalar case, the asymptotics for arithmetical means of the eigenvalues of block matrices with power behavior of eigenvalues of unperturbed operator and generalized Jacobi matrices with various number of non-zero off-diagonals.
\end{abstract}

Keywords: infinite tridiagonal block matrices, Jacobi matrices, the method of similar operators, eigenvalues, spectrum.

Mathematics Subjects Classifications: 47A75, 47B25, 47B36

\section{INTRODUCTION}

Let $\mathbb{C}^{m}, m \geqslant 1$, be an $m$-dimensional Euclidean space of vector columns equipped with the scalar product $(x, y)_{\mathbb{C}^{m}}=\sum_{i=1}^{m} x_{i} \overline{y_{i}}$, where complex numbers $x_{i}, y_{i}, i=1, \ldots, m$, are the coordinates of the vectors $x$ and $y$, respectively. By $l^{2}\left(\mathbb{N}, \mathbb{C}^{m}\right)$ we denote the Hilbert space of infinite sequences $u: \mathbb{N} \rightarrow \mathbb{C}^{m}, u=\left(u_{1}, u_{2}, \ldots\right), u_{n} \in \mathbb{C}^{m}, n \in \mathbb{N}$, equipped with the scalar product $(u, v)_{l^{2}\left(\mathbb{N}, \mathbb{C}^{m}\right)}=\sum_{n=1}^{\infty}\left(u_{n}, v_{n}\right)_{\mathbb{C}^{m}}$.

I.N. Braeutigam, D.M. Polyakov, Asymptotics of eigenvalues of infinite Block matrices.

(c) Braeutigam I.N., Polyakov D.M. 2019.

The work of the first author was financially supported by the Ministery of Education and Science of Russian Federation and DAAD (grant no. 1.12791.2018/12.2).

The reported study of the second author was funded by RFBR according to the research project 18-31-00205.

Submitted February 18, 2019. 
We consider an infinite tridiagonal block matrix

$$
\mathbf{J}:=\left(\begin{array}{ccccc}
\mathcal{A}_{1} & -\mathcal{B}_{1} & \mathcal{O} & \mathcal{O} & \ldots \\
-\widetilde{\mathcal{B}}_{1} & \mathcal{A}_{2} & -\mathcal{B}_{2} & \mathcal{O} & \ldots \\
\mathcal{O} & -\widetilde{\mathcal{B}}_{2} & \mathcal{A}_{3} & -\mathcal{B}_{3} & \ldots \\
\vdots & \vdots & \vdots & \vdots & \ddots
\end{array}\right)
$$

where $\mathcal{A}_{n}, \mathcal{B}_{n}, \widetilde{\mathcal{B}}_{n}, n \in \mathbb{N}$, are complex-valued matrices of size $m \times m, \mathcal{A}_{n}$ are self-adjoint matrices and $\mathcal{O}$ is the zero matrix. We note that in literature, the matrix $\mathbf{J}$ is also called an infinite tridiagonal matrix with matrix entries.

The matrix $\mathbf{J}$ defines a linear operator $L: D(L) \subset l^{2}\left(\mathbb{N}, \mathbb{C}^{m}\right) \rightarrow l^{2}\left(\mathbb{N}, \mathbb{C}^{m}\right)$ with the domain

$$
D(L)=\left\{u \in l^{2}\left(\mathbb{N}, \mathbb{C}^{m}\right): \mathbf{J} u \in l^{2}\left(\mathbb{N}, \mathbb{C}^{m}\right)\right\},
$$

acting by the formula

$$
(L u)_{n}=-\widetilde{\mathcal{B}}_{n-1} u_{n-1}+\mathcal{A}_{n} u_{n}-\mathcal{B}_{n} u_{n+1}, \quad n \in \mathbb{N},
$$

for $u \in D(L)$. We observe that $\widetilde{\mathcal{B}}_{0}=\mathcal{O}$ as $n=1$.

Hereafter we assume that the domain of the operator $L$ coincides with the domain of the operator $L_{0}: D\left(L_{0}\right) \subset l^{2}\left(\mathbb{N}, \mathbb{C}^{m}\right) \rightarrow l^{2}\left(\mathbb{N}, \mathbb{C}^{m}\right)$ acting by the formula $\left(L_{0} u\right)_{n}=\mathcal{A}_{n} u_{n}, n \in \mathbb{N}$, that is,

$$
D(L)=D\left(L_{0}\right)=\left\{u \in l^{2}\left(\mathbb{N}, \mathbb{C}^{m}\right): \sum_{n=1}^{\infty}\left\|\mathcal{A}_{n} u_{n}\right\|^{2}<\infty\right\} .
$$

We assume that all eigenvalue of the operator $L_{0}$ are simple. Denoting by $\sigma_{n}$ the spectra of the matrices $\mathcal{A}_{n}, n \in \mathbb{N}$, we have $\sigma_{n} \cap \sigma_{j}=\varnothing, n \neq j$, and we suppose that

$$
d_{n}=\min _{n \neq j} \operatorname{dist}\left(\sigma_{n}, \sigma_{j}\right) \rightarrow \infty \quad \text { as } n \rightarrow \infty .
$$

Moreover, we assume that the matrices $\mathcal{B}_{n}, \widetilde{\mathcal{B}}_{n}, n \in \mathbb{N}$, satisfy the following conditions:

$$
\begin{aligned}
& \sum_{n=1}^{\infty} \frac{\left\|\mathcal{B}_{n}\right\|^{2}+\left\|\widetilde{\mathcal{B}}_{n}\right\|^{2}}{\operatorname{dist}^{2}\left(\sigma_{n}, \sigma_{n+1}\right)}<\infty \\
& \sum_{n=1}^{\infty} \frac{\left\|\mathcal{B}_{n}\right\|^{2}\left\|\mathcal{B}_{n+1}\right\|^{2}}{\operatorname{dist}^{2}\left(\sigma_{n+2}, \sigma_{n+1}\right)}+\sum_{n=1}^{\infty} \frac{\left\|\widetilde{\mathcal{B}}_{n}\right\|^{2}\left\|\widetilde{\mathcal{B}}_{n+1}\right\|^{2}}{\operatorname{dist}^{2}\left(\sigma_{n}, \sigma_{n+1}\right)}+\sum_{n=1}^{\infty} \frac{\left\|\mathcal{B}_{n}\right\|^{2}\left\|\widetilde{\mathcal{B}}_{n}\right\|^{2}}{\operatorname{dist}^{2}\left(\sigma_{n}, \sigma_{n+1}\right)}<\infty
\end{aligned}
$$

We note that if in addition we assume that $\widetilde{\mathcal{B}}_{n}$ coincides with the adjoint of $\mathcal{B}_{n}$, then $\mathbf{J}$ becomes symmetric and is called a block Jacobi matrix or a Jacobi matrix with matrix entries.

Infinite tridiagonal matrices with scalar or matrix entries arise in various mathematical models. For instance, they are important objects in studying the moment problem (matrix moment problem) [1]-4], in describing the spectral properties of the differential Schrödinger and Dirac operators with point interactions [5]-[9] and the Hill operator with trigonometric potentials [10.

The classification of the spectrum of the self-adjoint operators generated by such matrices is among the main issues studied for infinite tridiagonal matrices. In particular, the problems related with finding the conditions for the entries of infinite tridiagonal matrices, under which the spectrum is discrete or continuous were considered in [11]-[13. The problem of finding the eigenvalues of infinite symmetric and non-symmetric tridiagonal matrices or discrete SturmLiouville operator attracted a lot of attention [14]-[21]. There are several approaches for solving this problem. In papers [14]-16], an asymptotics was found for the eigenvalues of various classes of Jacobi matrices by the method of successive diagonalization. In [16], apart of the mentioned approach, there was applied a method based on an abstract result by G.V. Rozenblum and an approach based on constructing analytic models. However, one of the main methods is 
that of finding the eigenvalue of self-adjoint or non-self-adjoint tridiagonal Jacobi matrices by approximating them by the eigenvalues of truncated (finite) tridiagonal matrices employed in [17]- 21].

The main aim of the present work is to find asymptotic formulae for the eigenvalues of the operator $L$. Our approach is the method of similar operators [22]-[25], which differs essentially from ones mentioned above. Earlier, this approach was applied for determining asymptotic behavior for the eigenvalues of various classes of differential operators with matrix coefficients [26]-[28] as well as of second order difference operators with a growing potential considered in the space $l^{2}(\mathbb{Z}, \mathbb{C})[29],[30$.

The paper is organized as follows. In Section 2 we provide the main concept on the method of similar operator. Section 3 is devoted to a preliminary similarity transform for the operator $L$ and obtaining auxiliary estimates. The main result is proved in Section 4 and in Section 5 we provide various examples.

\section{Method of Similar OPERATORS AND ABSTRACT SCHEME OF STUDYING}

In this section we briefly present the main ideas of the method of similar operators, for more details see [22]-25]. We also construct a scheme of studying of abstract operators, which are close to an original operator $L$ by their properties.

Let $\mathcal{H}$ be a complex separable Hilbert space, End $\mathcal{H}$ be the Banach algebra of linear bounded operators acting in $\mathcal{H}$.

Definition 1. Two linear operators $A_{i}: D\left(A_{i}\right) \subset \mathcal{H} \rightarrow \mathcal{H}, i=1,2$, are called similar if there exists a continuously invertible operator $U \in$ End $\mathcal{H}$ such that $A_{1} U x=U A_{2} x, x \in D\left(A_{2}\right)$, $U D\left(A_{2}\right)=D\left(A_{1}\right)$. The operator $U$ is called the operator of transformation of the operator $A_{1}$ into $A_{2}$.

The advantage of considering similar operator is that some of their spectral properties coincide [24, Lm. 1]. In particular, the spectra of similar operators coincide.

Let $A: D(A) \subset \mathcal{H} \rightarrow \mathcal{H}$ be a closed linear operator. By $\sigma(A)$ and $\rho(A)$ we denote its spectrum and resolvent set, while the symbol $\mathfrak{L}_{A}(\mathcal{H})$ stands for the Banach space of the operators acting in $\mathcal{H}$ and bounded relative to $A$. Then we consider a linear operator $B: D(B) \subset \mathcal{H} \rightarrow \mathcal{H}$. We say that $B$ belongs to the space $\mathfrak{L}_{A}(\mathcal{H})$ if $D(A) \subseteq D(B)$ and the quantity

$$
\|B\|_{A}=\inf \{C>0:\|B x\| \leqslant C(\|x\|+\|A x\|), x \in D(A)\}
$$

is finite. This quantity serves as a norm in $\mathfrak{L}_{A}(\mathcal{H})$. By the symbol $C$ various positive constants.

The main object of our study is the operator $A-B$. The operator $A$ is regarded as an unperturbed operator, while $B$ serves as a perturbation. Moreover, we assume that the spectral characteristics we are interesting in are known for the operator $A$. We however observe that in most cases there is no similarity between the operators $A$ and $A-B$. To overcome this obstacle, in the space $\mathfrak{L}_{A}(\mathcal{H})$ we choose a subspace $\mathfrak{U}$ such that the operators of form $A-\widetilde{B}$, where $\widetilde{B} \in \mathfrak{U}$, are of a simple structure and hence, these operators are simple enough for studying the spectral characteristics we are interesting in. If the operator $A-B$ is similar to the operator $A-\widetilde{B}$, according Definition 1, it possesses the same properties.

We proceed to the formulations of the main definitions and theorems of the method of similar operators.

Definition 2. Let $\mathfrak{U}$ be a linear subspace of the operators and $J: \mathfrak{U} \rightarrow \mathfrak{U}, \Gamma: \mathfrak{U} \rightarrow$ End $\mathcal{H}$ be transformers, that is, linear operators in the space of linear operators. A triple $(\mathfrak{U}, J, \Gamma)$ is called an admissible triple for the operator $A$, and $\mathfrak{U}$ is called a space of admissible perturbations if the following conditions hold:

1) $\mathfrak{U}$ is a Banach space with a norm $\|\cdot\|_{*}$ continuously embedded into $\mathfrak{L}_{A}(\mathcal{H})$;

2) $J$ and $\Gamma$ are continuous transformers and $J$ is a projector; 
3) $(\Gamma X) D(A) \subset D(A), A(\Gamma X)-(\Gamma X) A=X-J X$ for each $X \in \mathfrak{U}$ and $Y=\Gamma X$ is the unique solution of the equation

$$
A Y-Y A=X-J X
$$

satisfying the condition $J Y=O$, where $O$ is the zero operator;

4) $X(\Gamma Y),(\Gamma Y) X \in \mathfrak{U}$ for each $X, Y \in \mathfrak{U}$ and there exists a constant $\gamma>0$ such that $\|\Gamma\| \leqslant \gamma$ and $\max \left\{\|X(\Gamma Y)\|_{*},\|(\Gamma X) Y\|_{*}\right\} \leqslant \gamma\|X\|_{*}\|Y\|_{*} ;$

5) for each $X \in \mathfrak{U}$ and each $\varepsilon>0$ there exists a number $\lambda_{\varepsilon} \in \rho(A)$ such that $\left\|X\left(A-\lambda_{\varepsilon} I\right)^{-1}\right\|<$ $\varepsilon$.

We observe that while constructing an admissible triple for particular classes of the operators, as a space $\mathfrak{U}$, one chooses some convenient Banach or Hilbert space. The operator $J$ is constructed so that the final perturbing operator, an analogue of the operator $B$, has a rather simple structure. The definition of the operator $\Gamma$ is closely related with the definition of the transformation operator $U$, which arises in Definition 1. Properties 3) - 5) of the admissible triple are needed for solvability of some nonlinear equations arising while making similarity transformation. We note that a construction of the admissible tripe is possible via a non-unique way. The main criterions for its choice are the presence of needed properties of the involved transformers and the convenience of its usage.

We proceed to the main theorem on similarity.

Theorem 1. Let $(\mathfrak{U}, J, \Gamma)$ be an admissible triple for an operator $A$. If $B \in \mathfrak{U}$ and the condition

$$
\|J\|\|B\|_{*}\|\Gamma\|<\frac{1}{4}
$$

holds, then the operator $A-B$ is similar to the operator $A-J X_{*}$, where the operator $X_{*} \in \mathfrak{U}$ is a solution to the nonlinear operator equation

$$
X=B \Gamma X-(\Gamma X) J B-(\Gamma X) J(B \Gamma X)+B=\Phi(X)
$$

considered in the space $\mathfrak{U}$. This solution can be found by the simple iterations method letting $X_{0}=O, X_{1}=B$ and so forth. At that, the operator $\Phi: \mathfrak{U} \rightarrow \mathfrak{U}$ is contracting in the ball $\left\{X \in \mathfrak{U}:\|X-B\|_{*} \leqslant 3\|B\|_{*}\right\}$, and the similarity transformation of the operator $A-B$ into the operator $A-J X_{*}$ is made by the invertible operator $I+\Gamma X_{*} \in$ End $\mathcal{H}$.

The proof of this theorem can be found in [22, Thm. 1.5] and in [23, Thm. 19.2]. Condition (5) ensures the solvability of nonlinear equation (6). The form of this equation is directly related with the transformation operator $I+\Gamma X_{*}$. At that, conditions 3) -5 ) of Definition 2 ensures the invertibility of this operator and its invariance with respect to the domain of the operator $A$. Thus, the operators $A-B$ and $A-J X_{*}$ satisfy all properties of similar operators in Definition 1.

Now we apply the described general scheme to abstract operators, whose spectral properties coincide with the properties of the operator $L$.

As an unperturbed operator $A: D(A) \subset \mathcal{H} \rightarrow \mathcal{H}$ we choose a self-adjoint operator with a discrete spectrum, whose matrix representation has a block-diagonal form with entries $\mathcal{A}_{n}$ in an orthonormal basis $e_{n, i}, n \in \mathbb{N}, i=1,2, \ldots, m$, in the space $\mathcal{H}$. Assume that the operator $A$ possesses simple eigenvalues $\lambda_{n, i}, n \in \mathbb{N}, i=1,2, \ldots, m$. Then the spectrum $\sigma(A)$ of the operator $A$ can be represented as

$$
\sigma(A)=\cup_{n \in \mathbb{N}} \sigma_{n}
$$

where $\sigma_{n} \cap \sigma_{j}=\varnothing, n \neq j, n, j \in \mathbb{N}$, and each of the sets $\sigma_{n}$ consists of $m$ elements. Moreover, we shall assume that the spectrum of the operator $A$ satisfies condition (1). 
By the symbol $P_{n}, n \in \mathbb{N}$, we denote the Riesz projector constructed by the spectral set $\sigma_{n}$. For each $x \in \mathcal{H}$ we define it as follows:

$$
P_{n} x=\sum_{i=1}^{m}\left(x, e_{n, i}\right) e_{n, i} .
$$

Hereinafter by $\mathfrak{S}_{2}(\mathcal{H})$ we denote the ideal of the Hilbere-Schmidt operators with the norm $\|\cdot\|_{2}$, see [31, Ch. 3, Sect. 9]. To each operator $X \in \mathfrak{S}_{2}(\mathcal{H})$, we associate a block matrix $X=\left(X_{n j}\right), n, j \in \mathbb{N}$, formed by the operators $X_{n j}=P_{n} X P_{j}$. Since the projectors $P_{n}, n \in \mathbb{N}$, are orthonormal projectors, we define then norm in $\mathfrak{S}_{2}(\mathcal{H})$ by the formula

$$
\|X\|_{2}=\left(\sum_{n, j \in \mathbb{N}}\left\|P_{n} X P_{j}\right\|_{2}^{2}\right)^{\frac{1}{2}} .
$$

Now we proceed to constructing an admissible triple. As a space of admissible perturbations $\mathfrak{U}$ we choose the space $\mathfrak{S}_{2}(\mathcal{H})$.

We introduce a transformer $J: \mathfrak{S}_{2}(\mathcal{H}) \rightarrow \mathfrak{S}_{2}(\mathcal{H})$ as follows:

$$
J X=\sum_{j=1}^{\infty} P_{j} X P_{j}, \quad X \in \mathfrak{S}_{2}(\mathcal{H}) .
$$

For each $k \in \mathbb{N}$ we define a family of transformers $J_{k}$ by the formula:

$$
J_{k} X=J\left(X-P_{(k)} X P_{(k)}\right)+P_{(k)} X P_{(k)}=P_{(k)} X P_{(k)}+\sum_{j=k+1}^{\infty} P_{j} X P_{j},
$$

where $X \in \mathfrak{S}_{2}(\mathcal{H})$ and $P_{(k)}=\sum_{l=1}^{k} P_{l}$. Since $X$ belongs to the space $\mathfrak{S}_{2}(\mathcal{H})$, the series in $(8)$ and (9) are convergent.

Now we construct the operator $\Gamma: \mathfrak{S}_{2}(\mathcal{H}) \rightarrow \mathfrak{S}_{2}(\mathcal{H})$. In order to do this, we denote the operator $\Gamma X$ by $Y$ and we consider the block matrix $\left(Y_{n j}\right), n, j \in \mathbb{N}$, of this operator. We denote the restriction of the operator $A$ on the subspace $\mathcal{H}_{n}$ as $A \mid \mathcal{H}_{n}=\mathcal{A}_{n} I_{n}$, where $\mathcal{H}_{n}=\operatorname{Im} P_{n}, I_{n}$ is the identity mapping in $\mathcal{H}_{n}$. Then equation (4) in condition 3) of Definition 2 for matrix entries $Y_{n j}$ is written as

$$
\mathcal{A}_{n} Y_{n j}-Y_{n j} \mathcal{A}_{j}=X_{n j}, \quad n \neq j, \quad n, j \in \mathbb{N} .
$$

At that, the inequality

holds true.

$$
\left\|Y_{n j}\right\|_{2} \leqslant \frac{\left\|X_{n j}\right\|_{2}}{\operatorname{dist}\left(\sigma_{n}, \sigma_{j}\right)}, \quad Y_{n n}=\mathcal{O}
$$

We define the family of transformers $\Gamma_{k}, k \in \mathbb{N}$, as follows:

$$
\Gamma_{k} X=\Gamma X-\Gamma\left(P_{(k)} X P_{(k)}\right)=\Gamma X-P_{(k)}(\Gamma X) P_{(k)} .
$$

Remark 1. We note that under the above construction, the transformers $J_{k}$ and $\Gamma_{k}, k \in \mathbb{N}$, are obtained by cutting out a finite dimensional block of size $m k \times m k$ located in the left upper corner of the matrix representation of the operators $J$ and $\Gamma$. Therefore, they differ from the operators $J$ and $\Gamma$ by finite rank operators. Moreover, it is easy to show that $\|\Gamma\|_{2} \leqslant C$ and according condition (5), the norm $\|B\|_{2}$ should be small enough. The employing of the operators $J_{k}$ and $\Gamma_{k}$ allows us to omit this restriction for the operator $B$.

Remark 2. The definition of the operators $J_{k}$ and $\Gamma_{k}$ implies immediately the relations:

$$
\begin{aligned}
& \left(J_{k} X\right) P_{n}=P_{n}(J X) P_{n}=P_{n} X P_{n}, \quad \Gamma_{k}\left(P_{n} X P_{n}\right)=O, \\
& P_{n}\left(\left(J_{k} X\right) \Gamma_{k} Y\right) P_{n}=O
\end{aligned}
$$

for all elements $X, Y \in \mathfrak{S}_{2}(\mathcal{H})$. 
Thus, we have constructed the triple $\left(\mathfrak{S}_{2}(\mathcal{H}), J_{k}, \Gamma_{k}\right)$. To apply Theorem 1 , we need to show that this triple is admissible. The proof of this fact is given in the next lemma, in which we also provide an estimate allowing us to omit the restriction for the operator $B$ mentioned in Remark 1.

Lemma 1. For each $k \in \mathbb{N}$, the triple $\left(\mathfrak{S}_{2}(\mathcal{H}), J_{k}, \Gamma_{k}\right)$ is admissible for the operator $A$ and the constant $\gamma=\gamma(k)$ in Definition 2 satisfies the estimate:

$$
\left\|\Gamma_{k}\right\|_{2} \leqslant \gamma=d_{k}^{-1} .
$$

Proof. We are going to check all properties in Definition 2. The first property holds thanks to that of the space $\mathfrak{S}_{2}(\mathcal{H})$.

Let us check property 2). By the construction, the operators $J_{k}$ and $\Gamma_{k}$ are continuous transformers. In view of formula $(9)$, we have

$$
\left\|J_{k} X\right\|_{2}^{2} \leqslant\left\|P_{(k)} X P_{(k)}\right\|_{2}^{2}+\sum_{j=k+1}^{\infty}\left\|P_{j} X P_{j}\right\|_{2}^{2} \leqslant\|X\|_{2}^{2} .
$$

And if the matrix $X$ is block-diagonal, that is, $X=\sum_{j=1}^{\infty} P_{j} X P_{j}$, then $J_{k} X=X$. Hence, $\left\|J_{k} X\right\|_{2}=\|X\|_{2}$ and $\left\|J_{k}\right\|_{2}=1$.

The proof of the properties 3) and 5) follows the same scheme as [27, Thm. 5].

We proceed to proving property 4$)$. Let $X, Y \in \mathfrak{S}_{2}(\mathcal{H})$. Then in view of condition (1), the following estimates hold true:

$$
\begin{aligned}
\sum_{\substack{n, j=k+1 \\
n \neq j}}^{\infty}\left\|\left(X \Gamma_{k} Y\right)_{n j}\right\|_{2}^{2} & \leqslant\|X\|_{2}^{2} \sum_{\substack{n, j=k+1 \\
n \neq j}}^{\infty} \frac{\left\|Y_{n j}\right\|_{2}^{2}}{\operatorname{dist}^{2}\left(\sigma_{n}, \sigma_{j}\right)} \\
& \leqslant\left(\min _{\substack{n \neq j \\
n, j \geqslant k+1}} \operatorname{dist}\left(\sigma_{n}, \sigma_{j}\right)\right)^{-2}\|X\|_{2}^{2}\|Y\|_{2}^{2} \leqslant d_{k}^{-2}\|X\|_{2}^{2}\|Y\|_{2}^{2}<\infty .
\end{aligned}
$$

Therefore, $X \Gamma_{k} Y \in \mathfrak{S}_{2}(\mathcal{H})$. In the same way we prove that the operator $\left(\Gamma_{k} Y\right) X$ belongs to the space $\mathfrak{S}_{2}(\mathcal{H})$. Moreover, estimate 13 implies immediately inequality (12).

Thus, the triple $\left(\mathfrak{S}_{2}(\mathcal{H}), J_{k}, \Gamma_{k}\right)$ is an admissible one for the operator $A$.

Hereafter till the end of this section we assume that the perturbation $B$ belongs to the space $\mathfrak{S}_{2}(\mathcal{H})$. Then on the base of Lemma 1 and abstract Theorem 1, we can formulate the main similarity theorem for the considered operator $A-B$.

Theorem 2. Let a number $k \in \mathbb{N}$ be such that the condition

$$
\|B\|_{2}<\frac{d_{k}}{4}
$$

Then the operator $A-B$ is similar to the operator $A-J_{k} X_{*}$, where $X_{*} \in \mathfrak{S}_{2}(\mathcal{H})$ is a solution to a nonlinear equation

$$
X=B \Gamma_{k} X-\left(\Gamma_{k} X\right)\left(J_{k} B\right)-\left(\Gamma_{k} X\right) J_{k}\left(B \Gamma_{k} X\right)+B,
$$

which can be found by the method of simple iterations letting $X_{0}=O, X_{1}=B, \ldots$ The similarity transformation of the operator $A-B$ into the operator $A-J_{k} X_{*}$ is made by the operator $I+\Gamma_{k} X_{*}$, that is, the identity

$$
A-B=\left(I+\Gamma_{k} X_{*}\right)\left(A-J_{k} X_{*}\right)\left(I+\Gamma_{k} X_{*}\right)^{-1}
$$

holds true.

We observe that by (1), condition (14) of this theorem holds for a sufficiently large $k$.

Theorem 2 allows us to obtain an information about the spectrum of the operator $A-B$. 
Theorem 3. Let the assumptions of Theorem 2 be satisfied. Then the operator $A-B$ possesses a discrete spectrum coinciding with the spectrum of the operator

$$
A-J_{k} X_{*}=A-P_{(k)} X_{*} P_{(k)}-\sum_{n=k+1}^{\infty} P_{n} X_{*} P_{n} .
$$

The identities

$$
\sigma(A-B)=\sigma\left(A_{(k)}\right) \bigcup\left(\bigcup_{n \geqslant k+1} \sigma\left(A_{n}\right)\right)=\sigma_{(k)} \bigcup\left(\bigcup_{n \geqslant k+1} \sigma_{n}\right)
$$

hold, where the operator $A_{(k)}$ is the restriction of the operator $A-J_{k} X_{*}$ on an invariant subspace $\mathcal{H}_{(k)}=\operatorname{Im} P_{(k)}$ and $A_{n}$ is the restriction of the operator $A-J_{k} X_{*}$ on $\mathcal{H}_{n}=\operatorname{Im} P_{n}$. The sets $\sigma_{(k)}, \sigma_{n}, n \geqslant k+1$, are mutually disjoint.

Proof. Since the operator $A$ is an operator with a discrete spectrum and the operator $J_{k} X_{*}$ is bounded, the operator $A-J_{k} X_{*}$ is also an operator with a discrete spectrum. By Theorem 2 , the operators $A-B$ and $A-J_{k} X_{*}$ are similar. Therefore, the operator $A-B$ is also an operator with a discrete spectrum and the identity $\sigma(A-B)=\sigma\left(A-J_{k} X_{*}\right)$ holds true.

Representation (16) follows (9). Moreover, by Theorem 2 and the properties of similar operators, see, for instance, [25, Lm. 1], we conclude that the operator $A-J_{k} X_{*}$ of form (16) commutes with all projectors $P_{(k)}, P_{n}, n \geqslant k+1$. Hence, the subspaces $\mathcal{H}_{(k)}=\operatorname{Im} P_{(k)}$, $\mathcal{H}_{n}=\operatorname{Im} P_{n}, n \geqslant k+1$, are invariant for this operator. Since $A-J_{k} X_{*}$ has a discrete spectrum, for each $\lambda_{0} \in \sigma\left(A-J_{k} X_{*}\right)$, there exists and eigenvector $x_{0} \in D(A)$ such that $\left(A-J_{k} X_{*}\right) x_{0}=\lambda_{0} x_{0}$. Thus, the form of the operator $J_{k} X_{*}$ implies the identities

$$
A_{(k)} P_{(k)} x_{0}=\lambda_{0} P_{(k)} x_{0}, \quad A_{n} P_{n} x_{0}=\lambda_{0} P_{n} x_{0}, n \geqslant k+1,
$$

where $A_{(k)}$ is the restriction of the operator $A-J_{k} X_{*}$ on the invariant subspace $\mathcal{H}_{(k)}=\operatorname{Im} P_{(k)}$ and $A_{n}$ is the restriction of the operator $A-J_{k} X_{*}$ on $\mathcal{H}_{n}=\operatorname{Im} P_{n}$. Since the system of the projectors $P_{(k)}, P_{n}, n \geqslant k+1$, is a partition of the unity, it follows from (18) that at least one of the vectors $P_{(k)} x_{0}, P_{n} x_{0}, n \geqslant k+1$, is non-zero. Therefore, $\lambda_{0}$ is an eigenvalue of the corresponding operator in the family of the operators $A_{(k)}, A_{n}, n \geqslant k+1$. Thus, the right hand side of identity (17) is a subset of the set $\sigma\left(A-J_{k} X_{*}\right)=\sigma(A-B)$. The opposite inclusion is obvious. Hence, identity (17) holds true.

Now we are in position to formulate and prove the main theorem of the present section; this theorem is devoted to asymptotic formulae for the arithmetic means of the eigenvalues of the operator $A-B$.

Theorem 4. Let condition (14) hold and the spectrum of the operator $A-B$ can be represented as (17). Then the sets $\sigma_{n}, n \geqslant k+1$, contain at most $m$ elements and the arithmetic mean for each of these sets coincide with the arithmetic mean of the eigenvalues of the matrix

$$
\mathfrak{A}_{n}=\mathcal{A}_{n}-\mathfrak{B}_{n}+\mathfrak{C}_{n} .
$$

Here $\mathcal{A}_{n}$ is the nth block in the block-diagonal representation of the matrix $A, \mathfrak{B}_{n}$ is a matrix of size $m \times m$ with entries $\left(B e_{n, i}, e_{n, i}\right), i=1, \ldots, m$. The matrix $\mathfrak{C}_{n}$ satisfies the estimate

$$
\left\|\mathfrak{C}_{n}\right\| \leqslant \frac{2}{d_{n}}\left\|P_{n} B-P_{n} B P_{n}\right\|_{2}\left\|B P_{n}-P_{n} B P_{n}\right\|_{2}, n \geqslant \max \left\{k+1, n_{0}\right\}
$$

where $n_{0}$ is an index starting with the inequality holds: $\|B\|_{2} \leqslant \frac{d_{n}}{6}$.

Proof. We apply the projector $P_{n}$ to identity (15). In view of Remark 2, we obtain the identity

$$
P_{n} X_{*} P_{n}=P_{n} B P_{n}+P_{n}\left(B \Gamma_{k} X_{*}\right) P_{n} .
$$


We represent the operator $P_{n}\left(B \Gamma_{k} X_{*}\right) P_{n}$ as

$$
P_{n}\left(B \Gamma_{k} X_{*}\right) P_{n}=P_{n}\left(B-J_{k} B\right)\left(\Gamma_{k} X_{*}\right) P_{n}=\left(P_{n} B-P_{n} B P_{n}\right)\left(\Gamma_{k} X_{*}\right) P_{n} .
$$

Hence,

$$
\left\|P_{n}\left(B \Gamma_{k} X_{*}\right) P_{n}\right\|_{2} \leqslant\left\|P_{n} B-P_{n} B P_{n}\right\|_{2}\left\|\left(\Gamma_{k} X_{*}\right) P_{n}\right\|_{2} .
$$

Applying formulae (11), we obtain $\left(\Gamma_{k} X_{*}\right) P_{n}=\Gamma_{k}\left(X_{*}-J_{k} X_{*}\right) P_{n}, n \geqslant k+1$. Thus, the estimate

$$
\left\|\left(\Gamma_{k} X_{*}\right) P_{n}\right\|_{2} \leqslant \frac{\left\|X_{*} P_{n}-P_{n} X_{*} P_{n}\right\|_{2}}{d_{n}} .
$$

is true. In view of Remark 2, by (21) we get the relation:

$$
P_{n} X_{*} P_{n}=P_{n} B P_{n}+P_{n} B \Gamma_{k}\left(X_{*}-P_{n} X_{*} P_{n}\right) P_{n}, \quad n \geqslant k+1 .
$$

We apply the projector $P_{n}$ from the right to (15). This gives the identity:

$$
\begin{aligned}
X_{*} P_{n}-P_{n} X_{*} P_{n}= & B P_{n}-P_{n} B P_{n}+B \Gamma_{k}\left(X_{*}-P_{n} X_{*} P_{n}\right) P_{n} \\
& -\Gamma_{k}\left(X_{*}-P_{n} X_{*} P_{n}\right) P_{n} B P_{n}-P_{n} B \Gamma_{k}\left(X_{*}-P_{n} X_{*} P_{n}\right) P_{n} .
\end{aligned}
$$

Hence,

$$
\left\|X_{*} P_{n}-P_{n} X_{*} P_{n}\right\|_{2} \leqslant\left\|B P_{n}-P_{n} B P_{n}\right\|_{2}+\frac{3}{d_{n}}\|B\|_{2}\left\|X_{*} P_{n}-P_{n} X_{*} P_{n}\right\|_{2} .
$$

And therefore, for $n \in \mathbb{N}$ such that the inequality $\frac{3}{d_{n}}\|B\|_{2} \leqslant \frac{1}{2}$ holds, we obtain

$$
\left\|X_{*} P_{n}-P_{n} X_{*} P_{n}\right\|_{2} \leqslant 2\left\|B P_{n}-P_{n} B P_{n}\right\|_{2} \text {. }
$$

Thus,

$$
\left\|\left(\Gamma_{k} X_{*}\right) P_{n}\right\|_{2} \leqslant \frac{2\left\|B P_{n}-P_{n} B P_{n}\right\|_{2}}{d_{n}} .
$$

It follows from this estimate and $(22)$ that

$$
\left\|P_{n}\left(B \Gamma_{k} X_{*}\right) P_{n}\right\|_{2} \leqslant \frac{2}{d_{n}}\left\|P_{n} B-P_{n} B P_{n}\right\|_{2}\left\|B P_{n}-P_{n} B P_{n}\right\|_{2}
$$

for $n \geqslant \max \left\{k+1, n_{0}\right\}$, where $n_{0}$ is an index starting with which the inequality $\|B\|_{2} \leqslant \frac{d_{n}}{6}$ holds.

By considering the restriction of the operator $A-J_{k} X_{*}$ on $\operatorname{Im} P_{n}$ and taking into consideration identity (21), we obtain representation (19).

\section{PRELIMINARY SIMILARITY TRANSFORMATION OF OPERATOR $L$}

In this section we return back to studying the operator $L$ generated by an infinite block matrix J. As the space $\mathcal{H}_{m}$, the space $l^{2}\left(\mathbb{N}, \mathbb{C}^{m}\right)$ serves. As an unperturbed operator, we consider a self-adjoint operator $L_{0}: D\left(L_{0}\right) \subset \mathcal{H} \rightarrow \mathcal{H}$ generated by the Jacobi matrix

$$
\mathbf{A}:=\left(\begin{array}{ccccc}
\mathcal{A}_{1} & \mathcal{O} & \mathcal{O} & \mathcal{O} & \ldots \\
\mathcal{O} & \mathcal{A}_{2} & \mathcal{O} & \mathcal{O} & \ldots \\
\mathcal{O} & \mathcal{O} & \mathcal{A}_{3} & \mathcal{O} & \ldots \\
\vdots & \vdots & \vdots & \vdots & \ddots
\end{array}\right)
$$

on the domain $D\left(L_{0}\right)=\left\{u \in l^{2}\left(\mathbb{N}, \mathbb{C}^{m}\right): \mathbf{A} u \in l^{2}\left(\mathbb{N}, \mathbb{C}^{m}\right)\right\}$ and acting by the rule $\left(L_{0} u\right)_{n}=$ $\mathcal{A}_{n} u_{n}, n \in \mathbb{N}, u \in D\left(L_{0}\right)$.

We recall that the eigenvalues of the operator $L_{0}$ are simple. As above, by $\sigma_{n}$ we denote the spectrum of the matrix $\mathcal{A}_{n}$ and assume that condition (1) holds. Thus, the spectrum $\sigma\left(L_{0}\right)$ of the operator $L_{0}$ can be represented as

$$
\sigma\left(L_{0}\right)=\cup_{n \in \mathbb{N}} \sigma_{n}
$$

where $\sigma_{n} \cap \sigma_{j}=\varnothing, n \neq j, n, j \in \mathbb{N}$, and each of the sets $\sigma_{n}$ consists of $m$ entries. 
We consider a standard basis in the space $l^{2}\left(\mathbb{N}, \mathbb{C}^{m}\right)$, in which the operator $L_{0}$ is represented by the matrix A. Namely, it consists of the vectors

$$
e_{n, i}=\left\{\Delta_{n, j}^{i}\right\}_{j=1}^{\infty},
$$

where $n \in \mathbb{N}, i=1, \ldots, m, \Delta_{n, j}^{i}=(0,0, \ldots, 0)^{t} \in \mathbb{C}^{m}$ for $j \neq n, t$ denotes the transposition, $\Delta_{n, n}^{i}=\left(\delta_{1, i}, \delta_{2, i}, \ldots, \delta_{m, i}\right)^{t} \in \mathbb{C}^{m}$ and $\delta_{s, i}$ is the Kronecker delta.

As above, by the symbol $P_{n}, n \in \mathbb{N}$, we denote the Riesz projector constructed by the spectral set $\sigma_{n}$ and defined by formula (7).

As a perturbation $B$, we choose the operator generated by an infinite block matrix

$$
\mathbf{B}:=\left(\begin{array}{ccccc}
\mathcal{O} & \mathcal{B}_{1} & \mathcal{O} & \mathcal{O} & \ldots \\
\widetilde{\mathcal{B}}_{1} & \mathcal{O} & \mathcal{B}_{2} & \mathcal{O} & \ldots \\
\mathcal{O} & \widetilde{\mathcal{B}}_{2} & \mathcal{O} & \mathcal{B}_{3} & \ldots \\
\vdots & \vdots & \vdots & \vdots & \ddots
\end{array}\right)
$$

Direct calculations show that conditions (11)-(3) formulated in Introduction ensure the $L_{0^{-}}$ relative compactness of the operator $B$, for more details see [32, Ch.14]. And therefore, the operator $L_{0}-B$ has a discrete spectrum.

Since the operator $B$ does not belong to the space of admissible perturbations $\mathfrak{S}_{2}(\mathcal{H})$, we can not apply the abstract scheme constructed in Section 2. Because of this we need to make a preliminary similarity transformation of the operator $L_{0}-B$ in the operator $L_{0}-\widetilde{B}$, where $\widetilde{B}$ belongs to the space $\mathfrak{S}_{2}(\mathcal{H})$.

Remark 3. The form of the matrix $\mathbf{B}$ and the fact that the operator $J_{k}$ defined in (9) is a diagonalizable operator imply the identities $J_{k} B=O$ and $\left(\Gamma_{k} B\right) J_{k} B=O$.

The following lemma will allows to make a preliminary similarity transformation.

Lemma 2. There exists a number $q \in \mathbb{N}$ such that the operators $B, J_{q} B, \Gamma_{q} B$ satisfy the following conditions

(a) $\Gamma_{q} B \in$ End $\mathcal{H}$ and $\left\|\Gamma_{q} B\right\|_{2}<1$;

(b) $\left(\Gamma_{q} B\right) \mathrm{D}\left(L_{0}\right) \subset \mathrm{D}\left(L_{0}\right)$;

(c) $B \Gamma_{q} B,\left(\Gamma_{q} B\right) J_{q} B \in \mathfrak{S}_{2}(\mathcal{H})$;

(d) $L_{0}\left(\Gamma_{q} B\right) x-\left(\Gamma_{q} B\right) L_{0} x=B x-\left(J_{q} B\right) x, x \in \mathrm{D}\left(L_{0}\right)$;

(e) for each $\varepsilon>0$ there exists $\lambda_{\varepsilon} \in \rho\left(L_{0}\right)$ such that $\left\|B\left(L_{0}-\lambda_{\varepsilon} I\right)^{-1}\right\|<\varepsilon$.

Proof. Let us prove property $(a)$. By $b_{s i}^{l r}$ we denote the quantity $\left(B e_{r, i}, e_{l, s}\right)_{l^{2}\left(\mathbb{N}, \mathbb{C}^{m}\right)}$, then

$$
b_{s i}^{l r}= \begin{cases}0, & |r-l|>1, l=r, \\ \widetilde{b}_{s i}^{r}, & l=r+1, \\ b_{s i}^{r-1}, & l=r-1,\end{cases}
$$

where $\widetilde{b}_{s i}^{r}$ is an entry in the matrix $\widetilde{\mathcal{B}}_{r}$ and $b_{s i}^{r-1}$ is an entry of the matrix $\mathcal{B}_{r-1}, s, i=1, \ldots, m$.

Now we are going to show that $\Gamma_{q} B$ is a Hilbert-Schmidt operator. In order to do this, let us first establish that $\Gamma B$ belongs to $\mathfrak{S}_{2}(\mathcal{H})$. Condition (2) and formula (24) imply the estimate

$$
\begin{aligned}
\sum_{l, r=1}^{\infty} \sum_{s, i=1}^{m}\left|\left(\Gamma B e_{r, i}, e_{l, s}\right)_{l^{2}\left(\mathbb{N}, \mathbb{C}^{m}\right)}\right|^{2} & \leqslant \sum_{r=2}^{\infty} \sum_{s, i=1}^{m} \frac{\left|b_{s i}^{r-1}\right|^{2}}{\operatorname{dist}^{2}\left(\sigma_{r}, \sigma_{r-1}\right)}+\sum_{r=1}^{\infty} \sum_{s, i=1}^{m} \frac{\left|\widetilde{b}_{s i}^{r}\right|^{2}}{\operatorname{dist}^{2}\left(\sigma_{r}, \sigma_{r+1}\right)} \\
& =\sum_{r=2}^{\infty} \frac{\left\|\mathcal{B}_{r-1}\right\|^{2}}{\operatorname{dist}^{2}\left(\sigma_{r}, \sigma_{r-1}\right)}+\sum_{r=1}^{\infty} \frac{\left\|\widetilde{\mathcal{B}}_{r}\right\|^{2}}{\operatorname{dist}^{2}\left(\sigma_{r}, \sigma_{r+1}\right)} \\
& =\sum_{r=1}^{\infty} \frac{\left\|\mathcal{B}_{r}\right\|^{2}+\left\|\widetilde{\mathcal{B}}_{r}\right\|^{2}}{\operatorname{dist}^{2}\left(\sigma_{r}, \sigma_{r+1}\right)}<\infty
\end{aligned}
$$


Thus, $\Gamma B \in \mathfrak{S}_{2}(\mathcal{H})$. Since by formula $(10)$ the operators $\Gamma_{q} B, q \in \mathbb{N}$, differ from the operator $\Gamma B$ by a finite rank operator, then $\Gamma_{q} \bar{B}$ is also a Hilbert-Schmidt operator, that is, $\Gamma_{q} B \in$ $\mathfrak{S}_{2}(\mathcal{H}) \subset$ End $\mathcal{H}$. Moreover, it also follows from 10 that

$$
\lim _{q \rightarrow \infty}\left\|\Gamma_{q} B\right\|_{2}^{2}=\lim _{q \rightarrow \infty}\left\|\Gamma B-P_{(q)}(\Gamma B) P_{(q)}\right\|_{2}^{2}=\lim _{q \rightarrow \infty} \sum_{\substack{\max \{n, j\} \\ \geqslant q+1}}\left\|P_{n}\left(\Gamma_{q} B\right) P_{j}\right\|_{2}^{2}=0 .
$$

Thus, in view of Remark 2, we can choose a sufficiently large $q \in \mathbb{N}$, for which the inequality $\left\|\Gamma_{q} B\right\|_{2} \leqslant \frac{1}{2}<1$ holds.

To prove properties $(b)$ and $(d)$, it is sufficient to reproduce the arguing in the proof of Lemma 7 in [24].

We proceed to proving property $(c)$. Employing conditions (3) and (24), we obtain the following estimates

$$
\begin{aligned}
\sum_{l, r=1}^{\infty} \sum_{s, i=1}^{m} \mid & \left.\left(B \Gamma B e_{r, i}, e_{l, s}\right)_{l^{2}\left(\mathbb{N}, \mathbb{C}^{m}\right)}\right|^{2} \leqslant \sum_{r=3}^{\infty} \sum_{s, i=1}^{m} \frac{\left|\sum_{h=1}^{m} b_{s h}^{r-2} b_{h i}^{r-1}\right|^{2}}{\operatorname{dist}^{2}\left(\sigma_{r}, \sigma_{r-1}\right)} \\
& +\sum_{r=1}^{\infty} \sum_{s, i=1}^{m} \frac{\left|\sum_{h=1}^{m} \widetilde{b}_{s h}^{r+1} \widetilde{b}_{h i}^{r}\right|^{2}}{\operatorname{dist}^{2}\left(\sigma_{r}, \sigma_{r+1}\right)}+\sum_{r=2}^{\infty} \sum_{s, i=1}^{m}\left|\frac{\sum_{h=1}^{m} \widetilde{b}_{s h}^{r-1} b_{h i}^{r-1}}{\operatorname{dist}\left(\sigma_{r}, \sigma_{r-1}\right)}+\frac{\sum_{h=1}^{m} b_{s h}^{r} \widetilde{b}_{h i}^{r}}{\operatorname{dist}\left(\sigma_{r}, \sigma_{r+1}\right)}\right|^{2} \\
\leqslant & \sum_{r=3}^{\infty} \sum_{s, i=1}^{m} \frac{\left(\sum_{h=1}^{m}\left|b_{s h}^{r-2}\right|^{2}\right)\left(\sum_{h=1}^{m}\left|b_{h i}^{r-1}\right|^{2}\right)}{\operatorname{dist}^{2}\left(\sigma_{r}, \sigma_{r-1}\right)}+\sum_{r=1}^{\infty} \sum_{s, i=1}^{m} \frac{\left(\sum_{h=1}^{m}\left|\widetilde{b}_{s h}^{r+1}\right|^{2}\right)\left(\sum_{h=1}^{m}\left|\widetilde{b}_{h i}^{r}\right|^{2}\right)}{\operatorname{dist}^{2}\left(\sigma_{r}, \sigma_{r+1}\right)} \\
\leqslant & 2 \sum_{r=2}^{\infty} \sum_{s, i=1}^{m} \frac{\left(\sum_{h=1}^{m}\left|\widetilde{b}_{s h}^{r-1}\right|^{2}\right)\left(\sum_{h=1}^{m}\left|b_{h i}^{r-1}\right|^{2}\right)}{\operatorname{dist}^{2}\left(\sigma_{r}, \sigma_{r-1}\right)}+2 \sum_{r=2}^{\infty} \sum_{s, i=1}^{m} \frac{\left(\sum_{h=1}^{m}\left|b_{s h}^{r}\right|^{2}\right)\left(\sum_{h=1}^{m}\left|\widetilde{b}_{h i}^{r}\right|^{2}\right)}{\operatorname{dist}^{2}\left(\sigma_{r}, \sigma_{r+1}\right)} \\
= & \sum_{r=3}^{\infty} \frac{\left\|\mathcal{B}_{r-2}\right\|^{2}\left\|\mathcal{B}_{r-1}\right\|^{2}}{\operatorname{dist}^{2}\left(\sigma_{r}, \sigma_{r-1}\right)}+\sum_{r=1}^{\infty} \frac{\left\|\widetilde{\mathcal{B}}_{r+1}\right\|^{2}\left\|\widetilde{\mathcal{B}}_{r}\right\|^{2}}{\operatorname{dist}^{2}\left(\sigma_{r}, \sigma_{r+1}\right)} \\
& +2 \sum_{r=2}^{\infty} \frac{\left\|\widetilde{\mathcal{B}}_{r-1}\right\|^{2}\left\|\mathcal{B}_{r-1}\right\|^{2}}{\operatorname{dist}^{2}\left(\sigma_{r}, \sigma_{r-1}\right)}+2 \sum_{r=2}^{\infty} \frac{\left\|\mathcal{B}_{r}\right\|^{2}\left\|\widetilde{\mathcal{B}}_{r}\right\|^{2}}{\operatorname{dist}^{2}\left(\sigma_{r}, \sigma_{r+1}\right)} \\
\leqslant & C\left(\sum_{r=1}^{\infty} \frac{\left\|\mathcal{B}_{r}\right\|^{2}\left\|\mathcal{B}_{r+1}\right\|^{2}}{\operatorname{dist}^{2}\left(\sigma_{r+2}, \sigma_{r+1}\right)}+\sum_{r=1}^{\infty} \frac{\left\|\widetilde{\mathcal{B}}_{r}\right\|^{2}\left\|\widetilde{\mathcal{B}}_{r+1}\right\|^{2}}{\operatorname{dist}^{2}\left(\sigma_{r}, \sigma_{r+1}\right)}+\sum_{r=1}^{\infty} \frac{\left\|\mathcal{B}_{r}\right\|^{2}\left\|\widetilde{\mathcal{B}}_{r}\right\|^{2}}{\operatorname{dist}^{2}\left(\sigma_{r}, \sigma_{r+1}\right)}<\infty\right.
\end{aligned}
$$

This implies that the operator $B \Gamma B$ is a Hilbert-Schmidt operator and hence, $B \Gamma_{q} B \in \mathfrak{S}_{2}(\mathcal{H})$.

It follows from Remark 3 that $\left(\Gamma_{q} B\right) J_{q} B \in \mathfrak{S}_{2}(\mathcal{H})$.

Property $e$ ) is implied by the self-adjointness of the operator $L_{0}$, the relative $L_{0}$-compactness of the operator $B$ and [32, Lm 14.3].

The next lemma is devoted to additional estimates, which will be employed in the proof of the main theorem in the next section. We provide this lemma here since its proof involves the arguing used in checking properties $a$ ) and $c$ ) in Lemma 2.

Lemma 3. The following estimates hold:

$$
\left\|P_{n}\left(\Gamma_{q} B\right)\right\|_{2} \leqslant d_{n}^{-1}\left(\left\|\widetilde{\mathcal{B}}_{n-1}\right\|^{2}+\left\|\mathcal{B}_{n}\right\|^{2}\right)^{\frac{1}{2}}
$$




$$
\begin{aligned}
\left\|\left(\Gamma_{q} B\right) P_{n}\right\|_{2} \leqslant & d_{n}^{-1}\left(\left\|\mathcal{B}_{n-1}\right\|^{2}+\left\|\widetilde{\mathcal{B}}_{n}\right\|^{2}\right)^{\frac{1}{2}} \\
\left\|P_{n}\left(B \Gamma_{q} B\right)\right\|_{2} \leqslant & C d_{n}^{-1}\left(\left\|\widetilde{\mathcal{B}}_{n-1}\right\|^{2}\left\|\widetilde{\mathcal{B}}_{n-2}\right\|^{2}+\left\|\widetilde{\mathcal{B}}_{n-1}\right\|^{2}\left\|\mathcal{B}_{n-1}\right\|^{2}\right. \\
& \left.+\left\|\mathcal{B}_{n}\right\|^{2}\left\|\mathcal{B}_{n+1}\right\|^{2}+\left\|\widetilde{\mathcal{B}}_{n}\right\|^{2}\left\|\mathcal{B}_{n}\right\|^{2}\right)^{\frac{1}{2}} \\
\left\|\left(B \Gamma_{q} B\right) P_{n}\right\|_{2} \leqslant & C d_{n}^{-1}\left(\left\|\mathcal{B}_{n-2}\right\|^{2}\left\|\mathcal{B}_{n-1}\right\|^{2}+\left\|\widetilde{\mathcal{B}}_{n-1}\right\|^{2}\left\|\mathcal{B}_{n-1}\right\|^{2}\right. \\
& \left.+\left\|\widetilde{\mathcal{B}}_{n}\right\|^{2}\left\|\mathcal{B}_{n}\right\|^{2}+\left\|\widetilde{\mathcal{B}}_{n+1}\right\|^{2}\left\|\widetilde{\mathcal{B}}_{n}\right\|^{2}\right)^{\frac{1}{2}} \\
\left\|P_{n}\left(B \Gamma_{q} B\right) P_{n}\right\|_{2} \leqslant & C d_{n}^{-1}\left(\left\|\widetilde{\mathcal{B}}_{n-1}\right\|^{2}\left\|\mathcal{B}_{n-1}\right\|^{2}+\left\|\mathcal{B}_{n}\right\|^{2}\left\|\widetilde{\mathcal{B}}_{n}\right\|^{2}\right)^{\frac{1}{2}} .
\end{aligned}
$$

Proof. To prove estimates (27) and (28), in (25) we let $l=n$ and $r=n$. This yields:

$$
\begin{aligned}
& \left\|P_{n}\left(\Gamma_{q} B\right)\right\|_{2}^{2} \leqslant \frac{\left\|\widetilde{\mathcal{B}}_{n-1}\right\|^{2}}{\operatorname{dist}^{2}\left(\sigma_{n-1}, \sigma_{n}\right)}+\frac{\left\|\mathcal{B}_{n}\right\|^{2}}{\operatorname{dist}^{2}\left(\sigma_{n}, \sigma_{n+1}\right)} \leqslant d_{n}^{-2}\left(\left\|\widetilde{\mathcal{B}}_{n-1}\right\|^{2}+\left\|\mathcal{B}_{n}\right\|^{2}\right), \\
& \left\|\left(\Gamma_{q} B\right) P_{n}\right\|_{2}^{2} \leqslant \frac{\left\|\mathcal{B}_{n-1}\right\|^{2}}{\operatorname{dist}^{2}\left(\sigma_{n}, \sigma_{n-1}\right)}+\frac{\left\|\widetilde{\mathcal{B}}_{n}\right\|^{2}}{\operatorname{dist}^{2}\left(\sigma_{n}, \sigma_{n+1}\right)} \leqslant d_{n}^{-2}\left(\left\|\mathcal{B}_{n-1}\right\|^{2}+\left\|\widetilde{\mathcal{B}}_{n}\right\|^{2}\right) .
\end{aligned}
$$

To prove estimates (29) and (30), in 26) we let $l=n$ and $r=n$ :

$$
\begin{aligned}
\left\|P_{n}\left(B \Gamma_{q} B\right)\right\|_{2}^{2} & \leqslant \frac{\left\|\mathcal{B}_{n}\right\|^{2}\left\|\mathcal{B}_{n+1}\right\|^{2}}{\operatorname{dist}^{2}\left(\sigma_{n+2}, \sigma_{n+1}\right)}+2 \frac{\left\|\widetilde{\mathcal{B}}_{n-1}\right\|^{2}\left\|\mathcal{B}_{n-1}\right\|^{2}}{\operatorname{dist}^{2}\left(\sigma_{n}, \sigma_{n-1}\right)}+2 \frac{\left\|\widetilde{\mathcal{B}}_{n}\right\|^{2}\left\|\mathcal{B}_{n}\right\|^{2}}{\operatorname{dist}^{2}\left(\sigma_{n}, \sigma_{n+1}\right)}+\frac{\left\|\widetilde{\mathcal{B}}_{n-1}\right\|^{2}\left\|\widetilde{\mathcal{B}}_{n-2}\right\|^{2}}{\operatorname{dist}^{2}\left(\sigma_{n}, \sigma_{n-1}\right)} \\
& \leqslant 2 d_{n}^{-2}\left(\left\|\widetilde{\mathcal{B}}_{n-1}\right\|^{2}\left\|\widetilde{\mathcal{B}}_{n-2}\right\|^{2}+\left\|\widetilde{\mathcal{B}}_{n-1}\right\|^{2}\left\|\mathcal{B}_{n-1}\right\|^{2}+\left\|\widetilde{\mathcal{B}}_{n}\right\|^{2}\left\|\mathcal{B}_{n}\right\|^{2}+\left\|\mathcal{B}_{n}\right\|^{2}\left\|\mathcal{B}_{n+1}\right\|^{2}\right) \\
\left\|\left(B \Gamma_{q} B\right) P_{n}\right\|_{2}^{2} & \leqslant \frac{\left\|\mathcal{B}_{n-2}\right\|^{2}\left\|\mathcal{B}_{n-1}\right\|^{2}}{\operatorname{dist}^{2}\left(\sigma_{n}, \sigma_{n-1}\right)}+2 \frac{\left\|\widetilde{\mathcal{B}}_{n-1}\right\|^{2}\left\|\mathcal{B}_{n-1}\right\|^{2}}{\operatorname{dist}^{2}\left(\sigma_{n}, \sigma_{n-1}\right)}+2 \frac{\left\|\widetilde{\mathcal{B}}_{n}\right\|^{2}\left\|\mathcal{B}_{n}\right\|^{2}}{\operatorname{dist}^{2}\left(\sigma_{n}, \sigma_{n+1}\right)}+\frac{\left\|\widetilde{\mathcal{B}}_{n+1}\right\|^{2}\left\|\widetilde{\mathcal{B}}_{n}\right\|^{2}}{\operatorname{dist}^{2}\left(\sigma_{n}, \sigma_{n+1}\right)} \\
& \leqslant 2 d_{n}^{-2}\left(\left\|\mathcal{B}_{n-2}\right\|^{2}\left\|\mathcal{B}_{n-1}\right\|^{2}+\left\|\widetilde{\mathcal{B}}_{n-1}\right\|^{2}\left\|\mathcal{B}_{n-1}\right\|^{2}+\left\|\widetilde{\mathcal{B}}_{n}\right\|^{2}\left\|\mathcal{B}_{n}\right\|^{2}+\left\|\widetilde{\mathcal{B}}_{n}\right\|^{2}\left\|\widetilde{\mathcal{B}}_{n+1}\right\|^{2}\right) .
\end{aligned}
$$

Letting $l=n$ and $r=n$ in $(26)$, we obtain

$$
\begin{aligned}
\left\|P_{n}\left(B \Gamma_{q} B\right) P_{n}\right\|_{2}^{2} & \leqslant 2\left(\frac{\left\|\widetilde{\mathcal{B}}_{n-1}\right\|^{2}\left\|\mathcal{B}_{n-1}\right\|^{2}}{\operatorname{dist}^{2}\left(\sigma_{n}, \sigma_{n-1}\right)}+\frac{\left\|\mathcal{B}_{n}\right\|^{2}\left\|\widetilde{\mathcal{B}}_{n}\right\|^{2}}{\operatorname{dist}^{2}\left(\sigma_{n}, \sigma_{n+1}\right)}\right) \\
& \leqslant 2 d_{n}^{-2}\left(\left\|\widetilde{\mathcal{B}}_{n-1}\right\|^{2}\left\|\mathcal{B}_{n-1}\right\|^{2}+\left\|\mathcal{B}_{n}\right\|^{2}\left\|\widetilde{\mathcal{B}}_{n}\right\|^{2}\right) .
\end{aligned}
$$

The following theorem holds [25, Thm. 2], [24, Thm. 9].

Theorem 5. Under the assumptions of Lemma 2, the operator $L_{0}-B$ is similar to the operator $L_{0}-J B-\widetilde{B}$, where $\widetilde{B}=(I+\Gamma B)^{-1}(B \Gamma B-(\Gamma B) J B)$, and the identity holds:

$$
\left(L_{0}-B\right)(I+\Gamma B)=(I+\Gamma B)\left(L_{0}-J B-\widetilde{B}\right) .
$$

On the base of this theorem and Remark 3 we formulate the first similarity theorem. 
Theorem 6. Let a number $q \in \mathbb{N}$ be such that the condition

$$
\left\|\Gamma_{q} B\right\|_{2} \leqslant \frac{1}{2}
$$

is satisfied. Then the operator $L=L_{0}-B$ is similar to the operator $L_{0}-\widetilde{B}$, where $\widetilde{B}$ belongs to $\mathfrak{S}_{2}(\mathcal{H})$ and reads as

$$
\widetilde{B}=\left(I+\Gamma_{q} B\right)^{-1}\left(B \Gamma_{q} B\right)
$$

At that, the identity holds:

$$
\left(L_{0}-B\right)\left(I+\Gamma_{q} B\right)=\left(I+\Gamma_{q} B\right)\left(L_{0}-\widetilde{B}\right) .
$$

Formula (33) obviously implies the chain of identities:

$$
\widetilde{B}=\left(\sum_{j=0}^{\infty}(-1)^{j}\left(\Gamma_{q} B\right)^{j}\right)\left(B \Gamma_{q} B\right)=B \Gamma_{q} B-\left(\Gamma_{q} B\right)\left(I+\Gamma_{q} B\right)^{-1}\left(B \Gamma_{q} B\right) .
$$

Theorem 6 allows us to reduce the study of the operator $L$ to the study of the operator $L_{0}-\widetilde{B}$, where $\widetilde{B}$ already belongs to the space of admissible perturbations $\mathfrak{S}_{2}(\mathcal{H})$. Thus, the general theory of the method of similar operators and the constructions made in Section 2 are valid for the operator $L_{0}-\widetilde{B}$. Taking this into consideration, we formulate the second similarity theorem.

Theorem 7. There exists a number $k \in \mathbb{N}, k \geqslant q+1$, such that inequalities (14) and (32) hold. Then the operator $L$ is similar to the operator $L_{0}-J_{k} X_{*}$, where $X_{*}$ is a solution to a nonlinear equation

$$
X=\widetilde{B} \Gamma_{k} X-\left(\Gamma_{k} X\right)\left(J_{k} \widetilde{B}\right)-\left(\Gamma_{k} X\right) J_{k}\left(\widetilde{B} \Gamma_{k} X\right)+\widetilde{B}
$$

and the operator $\widetilde{B}$ is defined by formula 34 ).

\section{MAIN RESULT}

In this section we prove the main theorem on asymptotic formulae for the eigenvalues of the operator $L$. To prove this theorem, we employ an explicit matrix representation for the operator $J_{k}\left(B \Gamma_{k} B\right)$. Before proceeding to the proof, we make the following remark.

Remark 4. The matrix representation of the operator $J_{k}\left(B \Gamma_{k} B\right)$ is block-diagonal and its $n$th is represented as

$$
\mathcal{D}_{n}=\widetilde{\mathcal{B}}_{n-1} \mathcal{C}_{n-1}+\mathcal{B}_{n} \widetilde{\mathcal{C}}_{n}
$$

where the entries of the matrices $\mathcal{C}_{n-1}$ and $\widetilde{\mathcal{C}}_{n}$ read as

$$
c_{i j}^{n-1}=\frac{b_{i j}^{n-1}}{\lambda_{i}^{n-1}-\lambda_{j}^{n}}, \quad \widetilde{c}_{i j}^{n}=\frac{\widetilde{b}_{i j}^{n}}{\lambda_{i}^{n+1}-\lambda_{j}^{n}},
$$

and $\lambda_{i}^{n}$ is the ith eigenvalue of the matrix $\mathcal{A}_{n}, b_{i j}^{n-1}$ and $\widetilde{b}_{i j}^{n}$ are the entries of the matrices $\mathcal{B}_{n-1}$ and $\widetilde{\mathcal{B}}_{n}$.

Definition 3. Given a bounded matrix $\mathcal{A}$ acting in $\mathbb{C}^{m}$, the arithmetic mean of its eigenvalues is defined as

$$
\widehat{\lambda}=\frac{1}{m} \sum_{i=1}^{m} \lambda_{i}
$$

where $\lambda_{i}$ are the eigenvalues of the matrix $A$. 
Theorem 8. There exists a number $k \in \mathbb{N}$, for which the spectrum of the operator $L$ is represented as

$$
\sigma(L)=\sigma_{(k)} \cup\left(\cup_{n \geqslant k+1} \sigma_{n}\right),
$$

where $\sigma_{(k)}$ is a finite set, and $\sigma_{n}$ is at most m-point set. Each of the sets $\sigma_{n}$ coincides with the spectrum of the restriction of the operator $L$ on the subspace $\operatorname{Im} P_{n}$, and for the arithmetic mean of the eigenvalues $\widehat{\lambda}_{n}, n \geqslant k+1$, of the set $\sigma_{n}$ the asymptotic representation holds:

$$
\widehat{\lambda}_{n}=\frac{1}{m} \operatorname{tr} \mathcal{A}_{n}-\frac{1}{m} \operatorname{tr} \mathcal{D}_{n}+\beta_{n},
$$

or

$$
\widehat{\lambda}_{n}=\frac{1}{m} \sum_{i=1}^{m} \lambda_{n, i}-\frac{1}{m} \sum_{i=1}^{m} \mu_{n, i}+\beta_{n}
$$

where the sequence $\beta_{n}$ is defined in (43), $\operatorname{tr} \mathcal{A}_{n}, \operatorname{tr} \mathcal{D}_{n}$ and $\lambda_{n, i}, \mu_{n, i}, i=1,2, \ldots, m$, are respectively the traces and the eigenvalues of the matrices $\mathcal{A}_{n}$ and $\mathcal{D}_{n}$, while the matrix $\mathcal{D}_{n}$ is defined in (36).

Proof. By Theorem 7, the operator $L$ is similar to the operator $L_{0}-J_{k} X_{*}$, where $X_{*}$ is a solution to equation (35). Then, in view of Theorem 3 , the spectrum of the operator $L$ can be represented as

$$
\sigma(L)=\sigma\left(L_{0}-J_{k} X_{*}\right)=\sigma\left(A_{(k)}\right) \cup\left(\cup_{n \geqslant k+1} \sigma\left(A_{n}\right)\right)=\sigma_{(k)} \cup\left(\cup_{n \geqslant k+1} \sigma_{n}\right),
$$

where $A_{(k)}$ is the restriction of the operator $L_{0}-J_{k} X_{*}$ on $\operatorname{Im} P_{(k)}$ and $A_{n}$ is the restriction of the operator $L_{0}-J_{k} X_{*}$ on $\operatorname{Im} P_{n}$. Since the dimension of the space $\operatorname{Im} P_{(k)}$ is finite, the set $\sigma\left(A_{(k)}\right)=\sigma_{(k)}$ is finite. Therefore, representation (37) holds true.

We are going to prove formulae (38) and (39). By the construction of the transformers $J_{k}$ and $\Gamma_{k}$ we have $J_{k}\left(\Gamma_{k} X_{*}\right) J_{k} \widetilde{B}=O$. Taking this identity into consideration and employing formulae (33) and (35), we represent the operator $L_{0}-J_{k} X_{*}$ as

$$
\begin{aligned}
L_{0}-J_{k} X_{*} & =L_{0}-J_{k}\left(X_{*}-\widetilde{B}+\widetilde{B}\right)=L_{0}-J_{k} \widetilde{B}-J_{k}\left(X_{*}-\widetilde{B}\right) \\
& =L_{0}-J_{k}\left(B \Gamma_{k} B\right)-J_{k}\left(\widetilde{B} \Gamma_{k} X_{*}\right)+T,
\end{aligned}
$$

where the operator $T$ involves all remaining terms in the operator $\widetilde{B}$.

Now we employ Theorem 4 . Since the operator $\widetilde{B}$ belongs to the space of admissible perturbations $\mathfrak{S}_{2}(\mathcal{H})$, formula $(19)$ and estimate 20$)$ hold, where the operator $\widetilde{B}$ serves as the operator $B$.

Let us find an exact estimate in formula (20). Applying the operator $P_{n}$ from the left and right to (34) and taking into consideration the identity $J_{k} B=O$, we get:

$$
\begin{aligned}
& \widetilde{B} P_{n}-P_{n} \widetilde{B} P_{n}=\left(B \Gamma_{k} B\right) P_{n}-P_{n}\left(B \Gamma_{k} B\right) P_{n}+\left(P_{n}\left(\Gamma_{k} B\right)-\Gamma_{k} B\right)\left(I+\Gamma_{k} B\right)^{-1}\left(B \Gamma_{k} B\right) P_{n}, \\
& P_{n} \widetilde{B}-P_{n} \widetilde{B} P_{n}=P_{n}\left(B \Gamma_{k} B\right)-P_{n}\left(B \Gamma_{k} B\right) P_{n}+P_{n}\left(\Gamma_{k} B\right)\left(I+\Gamma_{k} B\right)^{-1}\left(\left(B \Gamma_{k} B\right) P_{n}-B \Gamma_{k} B\right) .
\end{aligned}
$$

We estimate the norm of both sides in the obtained identities:

$$
\begin{aligned}
\left\|\widetilde{B} P_{n}-P_{n} \widetilde{B} P_{n}\right\|_{2} \leqslant & \left\|\left(B \Gamma_{k} B\right) P_{n}\right\|_{2}+\left\|P_{n}\left(B \Gamma_{k} B\right) P_{n}\right\|_{2} \\
& +\frac{\left\|P_{n}\left(\Gamma_{k} B\right)\right\|_{2}+\left\|\Gamma_{k} B\right\|_{2}}{1-\left\|\Gamma_{k} B\right\|_{2}}\left\|\left(B \Gamma_{k} B\right) P_{n}\right\|_{2}
\end{aligned}
$$

and

$$
\begin{aligned}
\left\|P_{n} \widetilde{B}-P_{n} \widetilde{B} P_{n}\right\|_{2} \leqslant & \left\|P_{n}\left(B \Gamma_{k} B\right)\right\|_{2}+\left\|P_{n}\left(B \Gamma_{k} B\right) P_{n}\right\|_{2} \\
& +\frac{\left\|P_{n}\left(\Gamma_{k} B\right)\right\|_{2}}{1-\left\|\Gamma_{k} B\right\|_{2}}\left(\left\|\left(B \Gamma_{k} B\right)\right\|_{2}+\left\|\left(B \Gamma_{k} B\right) P_{n}\right\|_{2}\right) .
\end{aligned}
$$


Substituting estimates (27)-(31) into (40) and (41) and bearing in mind the inequality $\left\|\Gamma_{k} B\right\| \leqslant$ $\frac{1}{2}$, we obtain:

$$
\begin{aligned}
\left\|\widetilde{B} P_{n}-P_{n} \widetilde{B} P_{n}\right\|_{2}\left\|P_{n} \widetilde{B}-P_{n} \widetilde{B} P_{n}\right\|_{2} \leqslant \frac{C}{d_{n}^{2}}( & \left\|\mathcal{B}_{n-2}\right\|^{2}\left\|\mathcal{B}_{n-1}\right\|^{2}+\left\|\mathcal{B}_{n}\right\|^{2}\left\|\mathcal{B}_{n+1}\right\|^{2} \\
& +\left\|\widetilde{\mathcal{B}}_{n-1}\right\|^{2}\left\|\mathcal{B}_{n-1}\right\|^{2}+\left\|\widetilde{\mathcal{B}}_{n}\right\|^{2}\left\|\mathcal{B}_{n}\right\|^{2} \\
& \left.+\left\|\widetilde{\mathcal{B}}_{n-1}\right\|^{2}\left\|\widetilde{\mathcal{B}}_{n-2}\right\|^{2}+\left\|\widetilde{\mathcal{B}}_{n+1}\right\|^{2}\left\|\widetilde{\mathcal{B}}_{n}\right\|^{2}\right) .
\end{aligned}
$$

Hence, inequality (23) implies the estimate

$$
\left\|P_{n}\left(\widetilde{B} \Gamma_{k} X_{*}\right) P_{n}\right\|_{2} \leqslant C \beta_{n}
$$

where

$$
\begin{aligned}
\beta_{n}=d_{n}^{-3}( & \left\|\mathcal{B}_{n-2}\right\|^{2}\left\|\mathcal{B}_{n-1}\right\|^{2}+\left\|\mathcal{B}_{n}\right\|^{2}\left\|\mathcal{B}_{n+1}\right\|^{2}+\left\|\widetilde{\mathcal{B}}_{n-1}\right\|^{2}\left\|\mathcal{B}_{n-1}\right\|^{2} \\
& \left.+\left\|\widetilde{\mathcal{B}}_{n}\right\|^{2}\left\|\mathcal{B}_{n}\right\|^{2}+\left\|\widetilde{\mathcal{B}}_{n-1}\right\|^{2}\left\|\widetilde{\mathcal{B}}_{n-2}\right\|^{2}+\left\|\widetilde{\mathcal{B}}_{n+1}\right\|^{2}\left\|\widetilde{\mathcal{B}}_{n}\right\|^{2}\right) .
\end{aligned}
$$

The form of the operator $J_{k}\left(B \Gamma_{k} B\right)$, see formula (31), implies that the arithmetic means of its eigenvalues are of order $d_{n}^{-1}\left(\left\|\widetilde{\mathcal{B}}_{n-1} \mathcal{B}_{n-1}\right\|+\left\|\mathcal{B}_{n} \widetilde{\mathcal{B}}_{n}\right\|\right)$, that is, they are not involved into the remaining terms but are selected as a separate term in formula (39).

Since in a finite-dimensional space the spectral trace coincides with the matrix one, the arithmetic mean of the eigenvalues of the restriction of the operator $L_{0}-J_{k}\left(B \Gamma_{k} B\right)$ on the subspace $\operatorname{Im} P_{n}$ is determined as $\frac{1}{m} \operatorname{tr} \mathcal{A}_{n}-\frac{1}{m} \operatorname{tr} \mathcal{D}_{n}$ or respectively $\frac{1}{m} \sum_{i=1}^{m} \lambda_{n, i}-\frac{1}{m} \sum_{i=1}^{m} \mu_{n, i}$. Taking into consideration estimates $(23)$ and $(42)$, we arrive at formulae $(38)$ and $(39)$.

\section{EXAMPLES}

We first assume that the matrices $\mathcal{A}_{n}, \mathcal{B}_{n}, \widetilde{\mathcal{B}}_{n}$ are of size $1 \times 1$, that is, $\mathcal{A}_{n}:=a_{n}, \mathcal{B}_{n}:=b_{n}$, $\widetilde{\mathcal{B}}_{n}:=c_{n}$ and the matrix $\mathbf{J}$ is an infinite scalar tridiagonal matrix.

1. Following work [14], we assume that the entries of the matrix $\mathbf{J}$ read as

$$
a_{n}=n^{2}+c_{1} n+c_{2} n^{-1}+c_{3} n^{-2}+O\left(n^{-3}\right), \quad b_{n}=c_{n}=-g-p_{1} n^{-1}-p_{2} n^{-2}+O\left(n^{-3}\right),
$$

where $c_{j}, p_{j} \in \mathbb{R}, j=1,2,3, b_{n} \neq 0$. The operator $L$ generated by the matrix $\mathbf{J}$ has a discrete spectrum. Let us find the asymptotics for its eigenvalues.

It is obvious that the eigenvalues of the operator $L_{0}$ generated by the matrix $\mathbf{A}$, see Section 3 , satisfy the identity

$$
\lambda_{n}=n^{2}+c_{1} n+c_{2} n^{-1}+c_{3} n^{-2}+O\left(n^{-3}\right) .
$$

In order to be able to apply Theorem 8, we need to check conditions (2) and (3). It is obvious that the series

$$
\sum_{n=1}^{\infty} \frac{2\left(g+p_{1} n^{-1}+p_{2} n^{-2}+O\left(n^{-3}\right)\right)^{2}}{\left(\lambda_{n+1}-\lambda_{n}\right)^{2}}
$$

converges. Hence, condition (2) is satisfied. Let us check condition (3). The relations hold true:

$$
\begin{aligned}
& \sum_{n=1}^{\infty} \frac{b_{n}^{2} b_{n+1}^{2}}{\left(\lambda_{n+2}-\lambda_{n+1}\right)^{2}}+\sum_{n=1}^{\infty} \frac{b_{n}^{2} b_{n+1}^{2}}{\left(\lambda_{n}-\lambda_{n+1}\right)^{2}}+\sum_{n=1}^{\infty} \frac{b_{n}^{2} b_{n}^{2}}{\left(\lambda_{n}-\lambda_{n+1}\right)^{2}} \leqslant \\
& \leqslant C \sum_{n=1}^{\infty} \frac{g^{4}}{\left(2 n+1+c_{1}-c_{2} n^{-1}(n+1)^{-1}+O\left(n^{-3}\right)\right)^{2}} \leqslant C g^{4} \sum_{n=1}^{\infty} \frac{1}{4 n^{2}}<\infty
\end{aligned}
$$


Thus, we can apply Theorem 8 . Then the eigenvalues $\widetilde{\lambda}_{n}$ of the operator $L$ satisfy the following asymptotic representation:

$$
\widetilde{\lambda}_{n}=\lambda_{n}-\mu_{n}+O\left(n^{-3}\right),
$$

where

$$
\mu_{n}=\frac{b_{n-1}^{2}}{\lambda_{n-1}-\lambda_{n}}+\frac{b_{n}^{2}}{\lambda_{n+1}-\lambda_{n}},
$$

see (36). Substituting initial values into this expression, we arrive at a final asymptotic formula:

$$
\widetilde{\lambda}_{n}=n^{2}+c_{1} n+c_{2} n^{-1}+c_{3} n^{-2}+\frac{g^{2}}{2} n^{-2}+O\left(n^{-3}\right) .
$$

This formula agrees with Theorem 3.1 of work [14.

2. Assume that the entries of an infinite tridiagonal matrix $\mathbf{J}$ satisfy the following conditions:

a) $a_{n} \in \mathbb{R},\left|a_{n}\right| \rightarrow \infty$ as $n \rightarrow \infty$

b) $\left(\left|b_{n}\right|+\left|c_{n-1}\right|\right) / a_{n} \rightarrow 0$ as $n \rightarrow \infty$,

c) $b_{n} \neq 0, c_{n} \neq 0, n \in \mathbb{N}$.

Then the operator $L$ generated by $\mathbf{J}$ has a discrete spectrum and all eigenvalues of this operator have geometric multiplicity equal to 1 , see [10. If in addition we assume that all numbers $a_{n}, n \in \mathbb{N}$, are different and conditions (2) and (3) are satisfied, namely the following series converge:

d) $\sum_{n=1}^{\infty} \frac{\left|b_{n}\right|^{2}+\left|c_{n}\right|^{2}}{\left(a_{n+1}-a_{n}\right)^{2}}<\infty$

e) $\sum_{n=1}^{\infty} \frac{\left|b_{n}\right|^{2}\left|b_{n+1}\right|^{2}}{\left(a_{n+2}-a_{n+1}\right)^{2}}+\sum_{n=1}^{\infty} \frac{\left|c_{n}\right|^{2}\left|c_{n+1}\right|^{2}}{\left(a_{n+1}-a_{n}\right)^{2}}+\sum_{n=1}^{\infty} \frac{\left|b_{n}\right|^{2}\left|c_{n}\right|^{2}}{\left(a_{n+1}-a_{n}\right)^{2}}<\infty$.

Then, by applying Theorem 8 , we find asymptotic formulae for the eigenvalues of the operator $L$ :

where

$$
\lambda_{n}=a_{n}-\frac{c_{n-1} b_{n-1}}{a_{n-1}-a_{n}}-\frac{b_{n} c_{n}}{a_{n+1}-a_{n}}+\beta_{n}
$$

$$
\beta_{n}=O\left(\left(a_{n+1}-a_{n}\right)^{-3}\left(\sum_{s=0,2}\left(\left|b_{n-2+s}\right|^{2}\left|b_{n-1+s}\right|^{2}+\left|c_{n-1+s}\right|^{2}\left|c_{n-2+s}\right|^{2}\right)+\sum_{s=0,1}\left|c_{n-s}\right|^{2}\left|b_{n-s}\right|^{2}\right)\right) .
$$

We note that this example allows us to find the asymptotics for the eigenvalues of some Hill operators with a trigonometric potential, in particular, some Mathieu operators, see, for instance, 21].

In the following examples we consider infinite block tridiagonal matrices.

3. Suppose that the eigenvalues of the matrices $\mathcal{A}_{n}$ satisfy the representations $\lambda_{n, i}=c_{n, i} n^{\alpha}\left(1+\varepsilon_{n, i}\right), \alpha>0, c_{n, i}$ are some constants independent of $n$ and $\varepsilon_{n, i} \rightarrow 0$ as $n \rightarrow \infty$, $i=1, \ldots, m$. Let $\widetilde{\mathcal{B}}_{n}=\mathcal{B}_{n}^{*}$ and $\left\|\mathcal{B}_{n}\right\|=C n^{\beta}\left(1+\gamma_{n}\right), \gamma_{n} \rightarrow 0$ as $n \rightarrow \infty, \beta>0$. It is obvious that condition (2) holds as $\alpha>\beta+\frac{3}{2}$, and condition (3) is satisfied as $\alpha>2 \beta+\frac{3}{2}$. Then as $\alpha>2 \beta+\frac{3}{2}$ both conditions are satisfied. For these values of the parameters $\alpha$ and $\beta$, the operator $L$ with the domain

$$
D(L)=\left\{u \in l^{2}\left(\mathbb{N}, \mathbb{C}^{m}\right): \sum_{n=1}^{\infty}\left\|\mathcal{A}_{n} u_{n}\right\|^{2}<\infty\right\}
$$

generated by the Jacobi matrix $\mathbf{J}$ is a lower-semibounded self-adjoint operator in the space $l^{2}\left(\mathbb{N}, \mathbb{C}^{m}\right)$. The spectrum of the operator $L$ consists of its eigenvalues, and the asymptotics of their arithmetic means $\widehat{\lambda}_{n}$ is determined according Theorem 8 .

$$
\widehat{\lambda}_{n}=\frac{1}{m} n^{\alpha} \sum_{i=1}^{m} c_{n, i}\left(1+\varepsilon_{n, i}\right)-\frac{1}{m} \operatorname{tr} \mathcal{D}_{n}+\beta_{n},
$$


where the matrix $\mathcal{D}_{n}$ is defined in $(36)$, and $\beta_{n}=O\left(n^{4 \beta-3 \alpha+3}\right)$.

4. Assume that the eigenvalues of the matrices $\mathcal{A}_{n}$ are represented as $\lambda_{n, i}=n^{\alpha}\left(1+\varepsilon_{n, i}\right)$, $\varepsilon_{n, i} \rightarrow 0$ as $n \rightarrow \infty, i=1, \ldots, m$. Suppose that the matrices $\widetilde{\mathcal{B}}_{n}=\mathcal{B}_{n}^{*}$ are constant. Then conditions (2) and (3) hold as $\alpha>\frac{3}{2}$. At that, the spectrum of the operator $L$ consists of its eigenvalues and the asymptotics for their arithmetic means $\widehat{\lambda}_{n}$ is due to Theorem 8 .

$$
\widehat{\lambda}_{n}=n^{\alpha}\left(1+\tilde{\varepsilon}_{n, i}\right)-\frac{1}{m} n^{1-\alpha}\left(\sum_{h=1}^{m} \sum_{i=1}^{m} \frac{\overline{b_{h i}^{n-1}} b_{i h}^{n-1}}{d_{h, i}^{n-1}\left(1+\gamma_{h, i}^{n-1}\right)}+\sum_{h=1}^{m} \sum_{i=1}^{m} \frac{\overline{b_{h i}^{n}} b_{i h}^{n}}{d_{h, i}^{n}\left(1+\gamma_{h, i}^{n}\right)}\right)+\beta_{n},
$$

where $\overline{b_{h i}^{n}}, b_{i h}^{n}$ are the entries of the matrices $B_{n}^{*}$ and $B_{n}$, respectively, $\tilde{\varepsilon}_{n, i}, \gamma_{h, i}^{n-1}, \gamma_{h, i}^{n}$ are infinitesimal sequences and $d_{h, i}^{n-1}, d_{h, i}^{n}$ are constant numbers independent of $n$ and arising in the expressions $\lambda_{n-1, i}-\lambda_{n, i}$ and $\lambda_{n+1, i}-\lambda_{n, i}, \beta_{n}=O\left(n^{-3 \alpha+3}\right)$.

The next two examples are devoted to generalized Jacobi matrices. First we are going to show that such matrix can be represented as a block Jacobi matrix. Namely, we consider a generalized Jacobi matrix of dimension $2 m$ with real entries $c_{p, j}, p, j \in \mathbb{N}$, and $c_{p, j}=c_{j, p}$, $c_{p, j}=0$ as $|p-j|>m$ and $c_{p, p+m} \neq 0$, that is, this matrix has $2 m+1$ diagonals some of which can be zero except the last ones, see, for instance, [19], [33]. It is obvious that such generalized Jacobi matrix can be represented as an infinite block tridiagonal matrix $\mathbf{J}$ with matrix entries $\mathcal{A}_{n}, \mathcal{B}_{n}$ and $\mathcal{B}_{n}^{*}$ of size $m \times m$, which are of the form:

$$
\begin{gathered}
\mathcal{A}_{n}=\left(\begin{array}{cccc}
c_{n m, n m} & c_{n m, n m+1} & \ldots & c_{n m,(n+1) m-1} \\
c_{n m+1, n m} & c_{n m+1, n m+1} & \ldots & c_{n m+1,(n+1) m-1} \\
\vdots & \vdots & \ddots & \vdots \\
c_{(n+1) m-1, n m} & c_{(n+1) m-1, n m+1} & \ldots & c_{(n+1) m-1,(n+1) m-1}
\end{array}\right), \\
\mathcal{B}_{n}=\left(\begin{array}{cccc}
c_{n m,(n+1) m} & 0 & \ldots & 0 \\
c_{n m+1,(n+1) m} & c_{n m+1,(n+1) m+1} & \ldots & 0 \\
\vdots & \vdots & \ddots & \vdots \\
c_{(n+1) m-1,(n+1) m} & c_{(n+1) m-1,(n+1) m+1} & \ldots & c_{(n+1) m-1,(n+2) m-1}
\end{array}\right) .
\end{gathered}
$$

Assume that the operator $L$ generated by this generalized infinite Jacobi matrix has a discrete spectrum. Moreover, all eigenvalues $\lambda_{n, i}, i=1, \ldots, m, n \in \mathbb{N}$, of an unperturbed operator $L_{0}$ with the domain

$$
D\left(L_{0}\right)=\left\{u \in l^{2}\left(\mathbb{N}, \mathbb{C}^{m}\right): \sum_{n=1}^{\infty}\left\|\mathcal{A}_{n} u_{n}\right\|^{2}<\infty\right\}
$$

and acting by the formula $\left(L_{0} u\right)_{n}=\mathcal{A}_{n} u_{n}, n \in \mathbb{N}, u \in D\left(L_{0}\right)$, are different and the matrices $\mathcal{B}_{n}$ and $\mathcal{B}_{n}^{*}$ satisfy conditions (2) and (3). Then by Theorem 8 we can determine the arithmetic mean of the eigenvalues of the operator $L$.

5. Assume that the generalized Jacobi matrix has only three non-zero diagonals (the main one and two last off-diagonals) and its entries satisfy all aforementioned conditions. Then the arithmetic mean of the eigenvalues of the operator $L$ is determined by the formula

$$
\begin{aligned}
\widehat{\lambda}_{n}= & \frac{1}{m} \sum_{i=0}^{m-1} c_{n, m+i} \\
& -\frac{1}{m} \sum_{i=0}^{m-1}\left(\frac{c_{n m+i,(n-1) m+i}^{2}}{c_{(n-1) m,(n-1) m+i+1}-c_{n m, n m+i+1}}+\frac{c_{n m+i,(n+1) m+i}^{2}}{c_{(n+1) m,(n+1) m+i+1}-c_{n m, n m+i+1}}\right)+\beta_{n},
\end{aligned}
$$

where $\beta_{n}$ is defined in 43 . 
6. Assume that a generalized Jacobi matrix has five non-zero diagonals, then it can be represented as a tridiagonal Jacobi matrix with matrix entries of size $2 \times 2$, that is,

$$
\mathcal{A}_{n}=\left(\begin{array}{cc}
c_{2 n, 2 n} & c_{2 n, 2 n+1} \\
c_{2 n+1,2 n} & c_{2 n+1,2 n+1}
\end{array}\right), \quad \mathcal{B}_{n}=\left(\begin{array}{cc}
c_{2 n, 2(n+1)} & 0 \\
c_{2 n+1,2(n+1)} & c_{2 n+1,2(n+1)+1}
\end{array}\right) .
$$

Suppose that all aforementioned conditions are satisfied and the operator $L$ generated by a generalized Jacobi matrix has a discrete spectrum. It is obvious that the eigenvalues of the matrix $\mathcal{A}_{n}$ are given by the formula

$$
\lambda_{n, i}=\frac{1}{2}\left(c_{2 n, 2 n}+c_{2 n+1,2 n+1} \pm\left[\left(c_{2 n, 2 n}-c_{2 n+1,2 n+1}\right)^{2}+4 c_{2 n, 2 n+1} c_{2 n+1,2 n}\right]^{\frac{1}{2}}\right), \quad i=1,2 .
$$

Hence, according Theorem 8 , the arithmetic mean of the eigenvalues of the operator $L$ satisfies

$$
\widehat{\lambda}_{n}=\frac{1}{2}\left(c_{2 n, 2 n}+c_{2 n+1,2 n+1}\right)-\frac{1}{2} \sum_{i=0}^{1}\left(\frac{c_{2 n, 2(n-1)+i}^{2}}{\lambda_{n-1, i+1}-\lambda_{n, i+1}}+\frac{c_{2 n+i, 2(n+1)+i}^{2}}{\lambda_{n+1, i+1}-\lambda_{n, i+1}}\right)+\beta_{n},
$$

where $\beta_{n}$ is defined in 43 .

\section{BIBLIOGRAPHY}

1. M.G. Krein. Infinite J-matrices and matrix moment problem // Dokl. AN SSSR. 69:3, 125-128 (1949). (in Russian).

2. A.G. Kostyuchenko, K.A. Mirzoev. Three-term recurrence relations with matrix coefficients. The completely indefinite case // Matem. Zametki. 63:5, 709-716 (1998). [Math. Notes. 63:5, 624-630 (1998).]

3. B. Simon. The classical moment problem as a self-adjoint finite difference operator // Adv. Math. 137:1, 82-203 (1998).

4. A.J. Duran, P. Lopez-Rodrigez. The matrix moment problem // in "Margarita Mathematica en memoria de Jose Javier Guadalupe". L. Espanol and J. L. Varona, eds. Universidad de La Rioja, Logrono, 333-348 (2001).

5. A.S. Kostenko, M.M. Malamud, D.D. Natyagajlo. Matrix Schrödinger operator with $\delta$-Interactions // Matem. Zametki. 100:1, 59-77 (2016). [Math. Notes. 100:1, 49-65 (2016).]

6. K.A. Mirzoev, T.A. Safonova. on the deficiency index of the vector-valued Sturm-Liouville operator // Matem. Zametki. 99:2, 262-277 (2016). [Math. Notes. 99:2, 290-303 (2016).]

7. I.N. Braeutigam and K.A. Mirzoev. Deficiency indices of the operators generated by infinite Jacobi matrices with operator entries // Alg. Anal. 30:4, 1-26 (2018). [St. Petersburg Math. J. 30:4, 621638 (2019).]

8. V. Budyika, M. Malamud, A. Posilicano. Nonrelativistic Limit for $2 p \times 2 p$-Dirac operators with point interactions on a discrete set // Russ. J. Math. Phys. 24:4, 426-435 (2017).

9. V.S. Budyka, M.M. Malamud, A. Posilicano. To the spectral theory of one-dimensional matrix Dirac operators with point matrix interactions // Doklady AN. 479:2, 117-125 (2018). [Dokl. Math. 97:2, 115-121 (2018).]

10. P. Djakov, B. Mityagin. Simple and double eigenvalues of the Hill operator with a two-term potential // J. Appr. Theor. 135:1, 70-104 (2005).

11. P.A. Cojuhari, J. Janas. Discreteness of the spectrum for some unbounded Jacobi matrices // Acta Sci. Math. (Szeged). 73:3, 649-667 (2007).

12. P.A. Cojuhari. On the spectrum of a class of block jacobi matrices // in "Operator theory, structed matrices and dilations". Amer. Math. Soc., Providence, RI, 137-152 (2007).

13. S. Kupin, S. Naboko. On the instability of the essential spectrum for block Jacobi matrices // S. Constr Approx. 48:3, 473-500 (2018).

14. J. Janas, S. Naboko. Infinite Jacobi matrices with unbounded entries: Asymptotics of eigenvalues and the transformation operator approach// SIAM J. Math. Anal. 36:2, 643-658 (2004).

15. A. Boutet de Monvel, S. Naboko, L. Silva. The asymptotic behaviour of eigenvalues of modified Jaynes-Cummings model // Asympt. Anal. 47:3-4, 291-315 (2006). 
16. J.Janas, M. Malejki. Alternative approaches to asymptotic behavior of eigenvalues of some unbounded Jacobi matrices // J. Computat. Appl. Math. 200:1, 342-356 (2007).

17. M. Malejki. Eigenvalues for some complex infinite tridiagonal matrices // J. Adv. Math. Comp. Sci. 26:5, 1-9 (2018).

18. M. Malejki. Asymptotics of large eigenvalues for some discrete unbounded Jacobi matrices // Lin. Alg. Appl. 431:10, 1952-1970 (2009).

19. M. Malejki. Asymptotic behaviour and approximation of eigenvalues for unbounded block Jacobi matrices // Opuscula Math. 30:3, 311-330 (2010).

20. A. Boutet de Monvel, L. Zielinski. Approximation of eigenvalues for unbounded Jacobi matrices using finite submatrices // Cent. Eur. J. Math. 12:3, 445-463 (2010).

21. Y. Ikebe, N. Asai, Y. Miyazaki, D. Cai. The eigenvalue problem for infinite complex symmetric tridiagonal matrices with application // Lin. Alg. Appl. 241-243, 599-618 (1996).

22. A.G. Baskakov. Methods of abstract harmonic analysis in the perturbation of linear operators // Sibir. Matem. Zhurn. 24:1, 21-39 (1973). [Siber. Math. 24:1, 17-32 (1983)].

23. A.G. Baskakov. Harmonic analysis of linear operators Voronezh State Univ. Publ., Voronezh (1987). (in Russian).

24. A.G. Baskakov, D.M. Polyakov. The method of similar operators in the spectral analysis of the Hill operator with nonsmooth potential // Matem. Sborn. 208:1, 3-47 (2017). [Sb. Math. 208:1, 1-43 (2017).]

25. A.G. Baskakov, A.V. Derbushev, A.O. Shcherbakov. The method of similar operators in the spectral analysis of non-self-adjoint Dirac operators with non-smooth potentials // Izv. RAN. Ser. Matem. 75:3, 3-28 (2011). [Izv. Math. 75:3, 445-469 (2011).]

26. N.B. Uskova. On the spectral properties of a second-order differential operator with a matrix potential // Differ. Uravn. 52:5, 579-588 (2016). [Diff. Equat. 52:5, 557-567 (2016).]

27. N.B. Uskova. On spectral properties of Sturm-Liouville operator with matrix potential // Ufimskij Matem. Zhurn. 7:3, 88-99 (2015). [Ufa Math. J. 7:3, 84-94 (2015).]

28. I.N. Braeutigam, D.M. Polyakov. On the asymptotics of eigenvalues of a fourth-order differential operator with matrix coefficients // Differ. Uravn. 54:4, 458-474 (2018). [Diff. Equat. 54:4, 450467 (2018).]

29. G.V. Garkavenko, N.B. Uskova. Spectral analysis of a class of difference operators with growing potential // Vestnik VGU. Ser. Fiz. Matem. 3, 101-111 (2016). (in Russian).

30. G.V. Garkavenko, N.B. Uskova. The asymptotic of eigenvalues for difference operator with growing potential // Matem. Fiz. Komp. Model. 20:4, 6-17 (2017). (in Russian).

31. I.C. Gohberg, M.G. Krein. Introduction to the theory of linear nonselfadjoint operators in Hilbert space. Nauka, Moscow (1965). [Transl. Math. Monog. 18. Amer. Math. Soc. (1969).]

32. P.D. Hislop, I.M. Sigal. Introduction to spectral theory: with applications to Schrödinger operators. Springer, New York (1996).

33. A.L. Chistyakov. Defect indices of $J_{m}$-matrices and of differential operators with polynomial coefficients // Matem. Sborn. 85:4, 474-503 (1971). [Math. USSR-Sb. 14:4, 471-500 (1971).]

Irina Nikolaevna Brauetigam,

Fachhochschule Kiel,

Grüner Kamp,11,

24783, Osterrönfeld, Germany

E-mail: irinadolgih@rambler.ru

Dmitrii Mikhailovich Polyakov,

Southern Mathematical Institute

of Vladikavkaz Scientific Center of RAS,

Markus str. 22,

362027, Vladikavkaz, Russia

E-mail: dmitrypolyakow@mail.ru 